\title{
SUBMIT: Systemic therapy with or without up front surgery of the primary tumor in breast cancer patients with distant metastases at initial presentation
}

Jetske Ruiterkamp ${ }^{1}$, Adri C Voogd ${ }^{2}$, Vivianne CG Tjan-Heijnen ${ }^{3}$, Koop Bosscha ${ }^{4}$, Yvette M van der Linden ${ }^{5}$, Emiel JTh Rutgers ${ }^{6}$, Epie Boven ${ }^{7}$, Maurice JC van der Sangen ${ }^{8}$ and Miranda F Ernst ${ }^{3,9^{*}}$, for In collaboration with Dutch Breast Cancer Trialists' Group (BOOG)

\begin{abstract}
Background: Five percent of all patients with breast cancer have distant metastatic disease at initial presentation. Because metastatic breast cancer is considered to be an incurable disease, it is generally treated with a palliative intent. Recent non-randomized studies have demonstrated that (complete) resection of the primary tumor is associated with a significant improvement of the survival of patients with primary metastatic breast cancer. However, other studies have suggested that the claimed survival benefit by surgery may be caused by selection bias. Therefore, a randomized controlled trial will be performed to assess whether breast surgery in patients with primary distant metastatic breast cancer will improve the prognosis.

Design: Randomization will take place after the diagnosis of primary distant metastatic breast cancer. Patients will either be randomized to up front surgery of the breast tumor followed by systemic therapy or to systemic therapy, followed by delayed local treatment of the breast tumor if clinically indicated.

Patients with primary distant metastatic breast cancer, with no prior treatment of the breast cancer, who are 18 years or older and fit enough to undergo surgery and systemic therapy are eligible. Important exclusion criteria are: prior invasive breast cancer, surgical treatment or radiotherapy of this breast tumor before randomization, irresectable T4 tumor and synchronous bilateral breast cancer. The primary endpoint is 2-year survival. Quality of life and local tumor control are among the secondary endpoints. Based on the results of prior research it was calculated that 258 patients are needed in each treatment arm, assuming a power of $80 \%$. Total accrual time is expected to take 60 months. An interim analysis will be performed to assess any clinically significant safety concerns and to determine whether there is evidence that up front surgery is clinically or statistically inferior to systemic therapy with respect to the primary endpoint.
\end{abstract}

Discussion: The SUBMIT study is a randomized controlled trial that will provide evidence on whether or not surgery of the primary tumor in breast cancer patients with metastatic disease at initial presentation results in an improved survival.

Trial registration: NCT01392586.

Keywords: Primary metastatic breast cancer, surgery, randomised controlled trial

\footnotetext{
* Correspondence: m.ernst@jbz.nl

${ }^{3}$ Division of Medical Oncology, Department Internal Medicine, GROW-School for Oncology and Developmental Biology, Maastricht University Medical

Centre, Maastricht, The Netherlands

Full list of author information is available at the end of the article
} 


\section{Background}

In most western countries, around five percent of all patients with breast cancer have distant metastatic disease at initial presentation [1,2]. This accounts for 500 newly diagnosed patients each year in the Netherlands [1]. Because metastatic breast cancer is considered to be an incurable disease, the aim of the treatment for these patients is to provide palliation amongst others with systemic therapy. Usually, breast surgery is restricted to those patients in whom the breast tumor is symptomatic. The rationale behind this strategy is based on the fact that once distant metastases have occurred, (aggressive) local therapy provides no survival advantage.

Research on the effect of systemic therapy in women with metastatic disease has demonstrated that their prognosis has improved significantly during the last 10 to 15 years, primarily due to increased efficacy of chemotherapy and the introduction of targeted treatments [2-4]. Recent retrospective studies have demonstrated that resection of the breast tumor in patients with primary metastatic breast cancer is associated with a significant improvement of the prognosis (Table 1) [5-12]. The hazard ratios (HR) for overall survival in these studies ranged from 0,50 to 0,71 in favor of surgery of the breast tumor. Furthermore, in studies taking surgical resection margins into account, better survival was observed in patients with a primary breast lesion that had been removed with free surgical margins $[5,7,13]$. In the review of Ruiterkamp et al. a pooled HR of 0.65 (95\% confidence interval 0.59-0.72) was calculated for overall survival for surgery versus no surgery, in favor of surgery (Figure 1) [14]. Results of a stratified analysis, done by Rapiti et al. suggest a greater effect for surgery among women with only bone metastases at diagnosis [7].

In all these aforementioned studies, the decision to perform surgery could have been influenced by favorable prognostic factors, such as younger age, the presence of only one metastatic site or a good response to systemic therapy. To rule out the impact of potential confounders, most studies adjusted for age, tumor size, number and sites of metastases and hormone receptor status. In addition, some studies also adjusted for comorbidity or surgical margins.

More recent studies have investigated the role of additional confounding factors, such as timing of surgery, selection bias and coding errors, and came to the conclusion that the survival benefit seen in patients who were treated with a surgical resection of the primary tumor was not so strong or may have been explained by these confounders [15-18]. For example, in the study of Bafford et al., the benefit of surgery seemed confined to patients operated upon before diagnosis of metastatic disease and there was no survival advantage in patients who received an operation of the breast tumor after the diagnosis of the metastatic disease had taken place. This phenomenon was referred to as stage migration bias $[16,18]$. In a study by Leung et al., the benefit from surgery disappeared in the multivariate analysis when taking into account the use of chemotherapy [17]. Finally,

Table 1 Results of retrospective studies

\begin{tabular}{|c|c|c|c|c|c|c|c|c|c|}
\hline \multirow[t]{3}{*}{ Author } & \multirow[t]{3}{*}{ Year } & \multirow[t]{3}{*}{$\mathrm{Nr}$ of patients } & \multirow[t]{3}{*}{ Surgery (\%) } & \multirow[t]{3}{*}{$\mathrm{HR}$} & \multirow[t]{3}{*}{$95 \% \mathrm{Cl}$} & \multicolumn{4}{|c|}{ Median survival (months) } \\
\hline & & & & & & \multicolumn{3}{|c|}{ Surgery } & \multirow[t]{2}{*}{ No surgery } \\
\hline & & & & & & Not specified & Lumpec & Mastec & \\
\hline Khan [5] & 2002 & 16023 & 57 & 0.61 & $0.58-0.65$ & - & 27 & 32 & 19 \\
\hline Babiera [6] & 2006 & 224 & 37 & 0.50 & $0.21-1.19$ & - & & & - \\
\hline Rapiti [7] & 2006 & 300 & 42 & 0.60 & $0.4-1.0$ & -1 & & & - \\
\hline Fields [8] & 2007 & 409 & 46 & 0.53 & $0.42-0.67$ & 32 & & & 15 \\
\hline Gnerlich [9] & 2007 & 9734 & 47 & 0.63 & $0.60-0.66$ & 36 & & & 21 \\
\hline Blanchard [10] & 2008 & 395 & 61 & 0.71 & $0.56-0.91$ & 27 & & & 17 \\
\hline Cady $^{2}[15]$ & 2008 & 622 & 38 & - & - & - & & & - \\
\hline Bafford [16] & 2009 & 147 & 41 & 0.47 & - & 42 & & & 28 \\
\hline Ruiterkamp [11] & 2009 & 728 & 40 & 0.62 & $0.51-0.76$ & 31 & & & 14 \\
\hline Leung [17] & 2009 & 157 & 33 & - & - & 25 & & & 13 \\
\hline Neuman [12] & 2010 & 186 & 37 & 0.71 & $0.47-1.06$ & 40 & & & 33 \\
\hline Dominici $^{2}$ [18] & 2011 & 290 & 23 & 0.94 & $0.83-1.08$ & 42 & & & 41 \\
\hline
\end{tabular}




\begin{tabular}{|c|c|c|c|c|c|c|}
\hline Study or Subgroup & log[Risk Ratio] & SE & Weight & $\begin{array}{l}\text { RiskRatio } \\
\text { IV, Random, } 95 \% \mathrm{Cl}\end{array}$ & $\begin{array}{r}\text { Risk I } \\
\text { IV, Rando }\end{array}$ & $\begin{array}{l}\text { Ratio } \\
\mathrm{m}, 95 \% \mathrm{Cl}\end{array}$ \\
\hline Babiera 2005 & -0.69 & 0.44 & $1.3 \%$ & $0.50[0.21,1.19]$ & & F \\
\hline Bafford 2008 & -0.75 & 0.25 & $3.5 \%$ & $0.47[0.29,0.77]$ & & \\
\hline Blanchard 2007 & -0.34 & 0.124 & $9.4 \%$ & $0.71[0.56,0.91]$ & 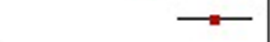 & \\
\hline Cady 2008 & 0 & 0 & & Not estimable & & \\
\hline Fields 2007 & -0.63 & 0.119 & $9.8 \%$ & $0.53[0.42,0.67]$ & - & \\
\hline Gnerlich 2007 & -0.48 & 0.029 & $19.0 \%$ & $0.62[0.58,0.65]$ & $=$ & \\
\hline Khan 2002 (1) & -0.49 & 0.029 & $19.0 \%$ & $0.61[0.58,0.65]$ & $=$ & \\
\hline Khan 2002 (2) & -0.29 & 0.027 & $19.1 \%$ & $0.75[0.71,0.79]$ & - & \\
\hline Leung 2009 & 0 & 0 & & Not estimable & & \\
\hline Rapiti 2006 (1) & -0.51 & 0.234 & $3.9 \%$ & $0.60[0.38,0.95]$ & & \\
\hline Rapiti $2006(2)$ & 0.26 & 0.246 & $3.6 \%$ & $1.30[0.80,2.10]$ & & \\
\hline Ruiterkamp 2009 & -0.48 & 0.102 & $11.3 \%$ & $0.62[0.51,0.76]$ & $\varpi$ & \\
\hline Total $(95 \% \mathrm{Cl})$ & & & $100.0 \%$ & $0.65[0.59,0.72]$ & & \\
\hline \multicolumn{5}{|c|}{$\begin{array}{l}\text { Heterogeneity: } \operatorname{Tau}^{2}=0.01 ; \mathrm{Chi}^{2}=47.04, \mathrm{df}=9(\mathrm{P}<0.00001) ; I^{2}=81 \% \\
\text { Test for overall effect: } Z=8.38(\mathrm{P}<0.00001)\end{array}$} & $\begin{array}{l}1 \\
0.2 \quad 0.5 \\
\text { Favours surgery }\end{array}$ & $\begin{array}{cc} & 1 \\
\text { Favours no surgery }\end{array}$ \\
\hline
\end{tabular}

in a study by Cady et al. coding errors in the retrospectively collected dataset were found to explain part of survival advantage [15].

Given the nature of retrospective analysis, it is not possible to provide a definite answer to the question whether surgical therapy of the breast tumor indeed affects overall survival. Therefore, in the Netherlands a randomized controlled trial (RCT) has been initiated and will start recruiting in the second half of 2011. This study is called, the SUBMIT trial, an acronym for 'Systemic therapy with or without $\mathbf{U p}$ front surgery of the primary tumor in Breast cancer patients with distant Metastases at Initial presenTation'. In the current paper we will present the design of this trial.

\section{Design}

The aim of the SUBMIT study is to investigate the effect of surgery of the primary tumor in breast cancer patients with distant metastatic disease at initial diagnosis. After diagnosis of primary distant metastatic breast cancer, patients will be randomly allocated in two groups: A. Up front breast surgery followed by systemic therapy; B. Systemic therapy potentially followed by delayed local treatment of the breast tumor (Figure 2).

\section{Patient selection criteria}

The eligibility criteria for the study are:

- primary distant metastatic breast cancer (M1); metastases diagnosed
- within one month after the diagnosis of the breast cancer

- an anticipated survival of at least 6 months;

- a histologically proven diagnosis of the breast tumor;

- a known hormonal and HER2Neu status;

- TNM classification: T1-T3, resectable T4 status and NO-N3;

- performance status and comorbidity should allow surgery and/or systemic therapy;

- age $\geq 18$ years;

- written informed consent.

Exclusion criteria are:

- a primary invasive breast cancer in the medical history;

- another malignancy within the last 10 years, besides basal cell carcinoma of the skin or early stage cervical cancer;

- surgical treatment and/or radiotherapy of the breast tumor before randomization;

- irresectable T4 breast tumor;

- synchronous bilateral breast cancer.

\section{Randomization}

Centralized randomization will take place immediately after the diagnosis of primary distant metastatic breast cancer, using a computer-generated randomization list. 


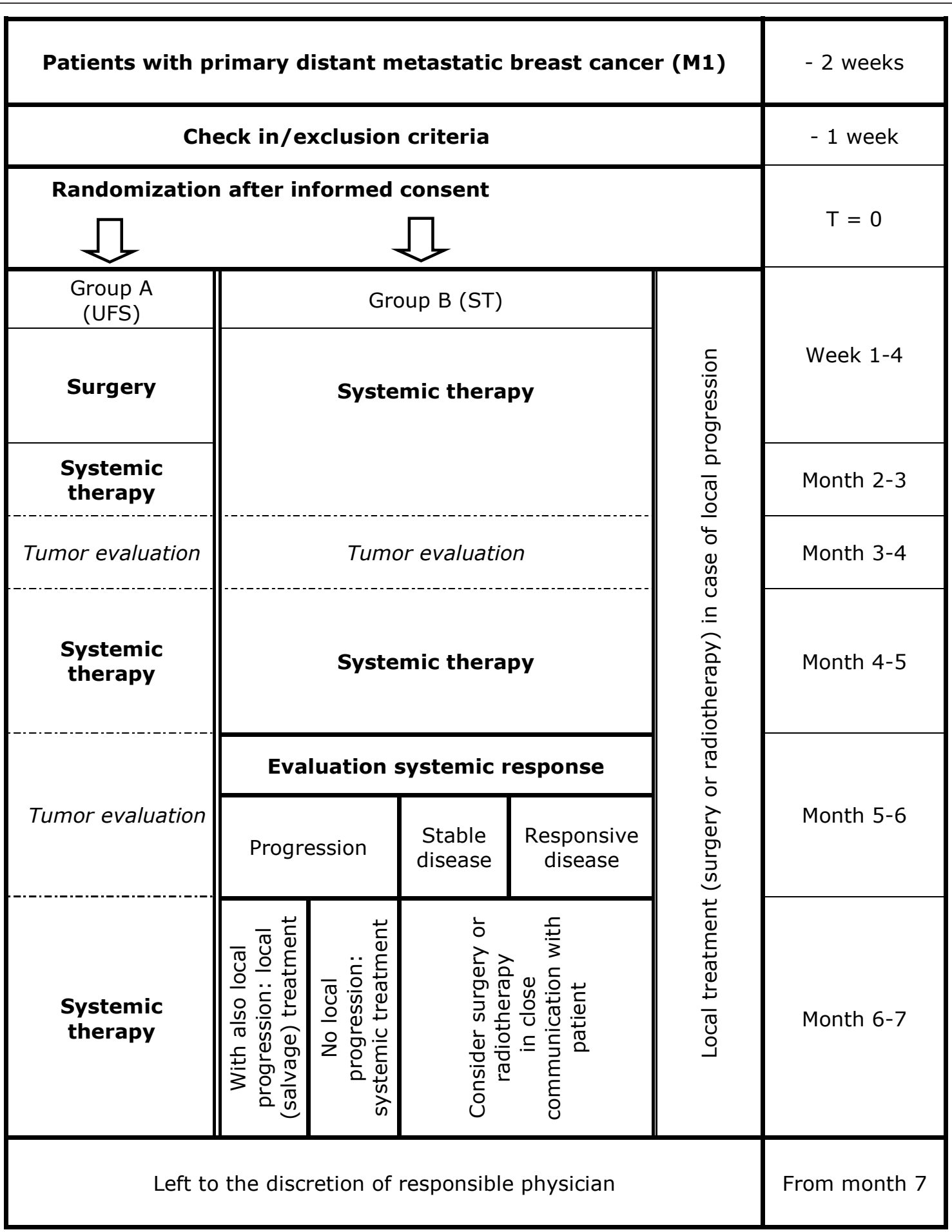

Figure 2 Study design.

Patients either randomize for up front surgery of the breast tumor followed by systemic therapy or for systemic therapy possibly followed by local treatment of the breast tumor if clinically indicated.
The randomization will be balanced by minimization, according to the minimization algorithm of Pocock and Simon [19], for: centre, age (18-49, 50-69, $\geq 70$ years), dominant location of distant metastases (bone, soft 
tissue (lymph nodes and subcutaneous metastases) and visceral metastases), hormonal receptor status (ER and/ or PR positive or both negative) and HER2Neu status (overexpression yes or no).

Randomization will be performed centrally, using a website with electronic data-entry to check treatment eligibility.

\section{Hypotheses}

The following three hypotheses will be tested:

1. Up front breast surgery in patients with primary distant metastatic breast cancer will result in a significant improvement of the 2-year survival compared to the survival achieved with palliative systemic therapy followed by delayed local treatment or systemic therapy alone.

2. Local tumor control in these patients will be superior in case of up front breast surgery compared to patients who receive systemic treatment with delayed local therapy or systemic therapy alone.

3. Better local control, by the use of up front breast surgery, results in a better quality of life in patients with Stage IV breast cancer, compared to patients who receive systemic treatment with delayed local therapy or systemic therapy alone.

\section{Ethical approval}

The study is approved by the one of the Dutch Medical Ethics Committees (METOPP; Medisch-Ethische Toetsing Onderzoek Patiënten en Proefpersonen, Tilburg). The project number is NL30331.028.11. The METOPP is an Ethical Review Committee according to the Dutch Medical Research Involving Human Subjects Act (WMO: Wet Medisch-Wetenschappelijk Onderzoek met Mensen). This study will be carried out in compliance with the Helsinki Declaration [20].

\section{Settings and location}

The study is a multicenter randomized controlled trial, which will be conducted in the Netherlands. Both academic and regional hospitals are allowed to participate, and already 25 centers are willing to participate.

\section{Treatment options}

During the study patients can be treated with surgery of the primary tumor (depending on randomization), systemic therapy and/or locoregional radiotherapy. The different treatment modalities are described below.

\section{Surgery of the primary tumor and axillary lymph node dissection}

Patients who are randomized to up front surgery of the breast tumor will receive a lumpectomy or mastectomy depending on patient and tumor characteristics. Both types of surgery may be conducted as long as the intention is a complete resection of the primary tumor, which is defined as having succeeded in obtaining free resection margins for the invasive component. In case of the presence of involved margins (more than focally) there are three options:

- to perform a re-excision or mastectomy (preferred options in up front surgery group);

- to treat the patient with locoregional radiotherapy;

- to accept that a non-radical resection has been performed.

What to decide belongs to the responsibility of the treating physician; he or she is not bound by restrictions in the protocol.

Also the choice to perform an axillary lymph node dissection is left to the discretion of the treating physician, but is highly recommended if palpable and/or tumor positive lymph nodes in the axillary region are present.

If patients are randomized for systemic therapy, they may still be candidates for surgery of the primary tumor. In case of local progression breast surgery is allowed at any time to gain local control, but preferentially not within the initial 5-6 months of first-line systemic therapy. Surgery for this group is primary indicated when the treating physician beliefs the tumor may cause wound problems in near future, despite use of systemic therapy.

\section{Locoregional radiotherapy}

There are two situations where radiotherapy to the breast or chest wall should be considered. First after an irradical lumpectomy or mastectomy. Irradicality in this trial is defined as involved margins for invasive breast cancer. The second situation is in case of local progression in patients in group B where a non invasive local therapy with radiotherapy is a treatment option instead of surgery. In case of clinically positive axillary lymph nodes, radiotherapy may be a viable treatment option instead of surgery as well. In both situations a hypofractionated regimen is mandatory without too much delay in systemic therapy, if applicable. Of note,-if considered indicated-radiotherapy can be postponed after first-line chemotherapy has been delivered. Concurrent radiotherapy and chemotherapy is not allowed because of expected excessive toxicity.

Radiotherapy may also be indicated for distant metastases, such als painful bone metastases or brain metastases.

\section{Systemic therapy}

Patients will be treated with systemic therapy according to the guidelines from the NAtional Breast cancer 
Organization of the Netherlands (NABON) for treatment of breast cancer. The choice of initial chemotherapy, immunotherapy and endocrine therapy dependsamong others-on ER and PR and HER2Neu status, dominant site of distant metastases, age, performance status and comorbidity; but may also depend on the chance to reach a complete remission for example in minimal metastatic disease. In patients with a hormone receptor positive tumor, hormonal treatment in indicated. In case of rapid progression, chemotherapy is the treatment of choice. In HER2Neu positive disease and the use of an aromatase inhibitor, it is possible to add HER2Neu targeted therapy. Chemotherapy is offered to patients with hormone receptor negative status, with extensive and fast growing visceral metastases and with severe cytopenia. For this study we advice to use at least an anthracycline, a taxane and capecitabine for the first 2-3 lines of treatment. The order is left to the discretion of the treating physician, if necessary in combination with targeted therapy, such as bevacizumab or HER2Neu targeted therapy, according to local practice. Patients with HER2Neu overexpression, can be treated with a combination of trastuzumab and taxanes as firstline chemotherapy. At first line treatment one may also choose for anthracycline containing chemotherapy, without HER2Neu targeted therapy. During the following lines of chemotherapy, continuing HER2Neu blocking with trastuzumab or lapatinib is advised.

\section{Primary and secondary outcome measures}

The primary endpoint is the two-year survival. This is defined as the percentage of patients who survive two years after randomization. Secondary endpoints are quality of life, overall survival, number of unplanned local therapies, i.e. surgery or radiotherapy, number of axillary lymph node dissections or axillary radiotherapy, determination of pathological resection margin (margin status) in patients treated by surgery of the primary tumor and type of chemotherapy, immunotherapy and endocrine therapy and number of regimens of systemic therapy during the first 2 years.

\section{Statistics}

\section{Sample size calculation}

In a previous, retrospective, study performed in the south of the Netherlands, the median survival of patients with stage IV breast cancer who had surgery was 31 months, as compared to 14 months for patients who did not have surgery $(P<0.0001)$ [11]. In a multivariable Cox regression analysis, adjusting for age, period of diagnosis, T-classification, number of metastatic sites, co-morbidity, use of locoregional radiotherapy and use of systemic therapy, the HR of breast surgery for overall mortality in this study was 0.62 (95\% confidence interval (CI) 0.51-0.76). In a recently published metaanalysis, including the results of 9 retrospective studies, the pooled HR for overall mortality was 0.65 (95\% CI 0.59-0.72) [14].

We are planning a randomized controlled trial with an equal number of patients in both treatment arms, an accrual interval of 60 months, and additional follow-up after the accrual interval of 18 months. We assume that (as a result of more effective systemic treatments) the median survival time of the patients without surgery has improved to 18 months since 2004, which was the last period of diagnosis included in the retrospective study based on the data from the Eindhoven Cancer Registry [1]. If the true hazard ratio (relative risk) of the patients with upfront surgery relative to the patients with immediate start of systemic therapy is 0.76 (the upper boundary of the $95 \%$ CI of the retrospective study, performed in the south of the Netherlands by Ruiterkamp et al. [11]), we will need to study 248 patients with up front surgery and 248 without up front surgery to be able to reject the null hypothesis that the survival curves are equal with a probability (power) of 0.80 . The Type I error probability associated with this test of this null hypothesis is 0.05 . An additional number of 20 patients will be included to account for loss to follow-up and/or exclusion after randomization because of violation of the eligibility criteria; so total accrual consists of 516 patients.

\section{Data analysis}

Investigators will enter the information required by the protocol into the Case Report Forms (CRFs). The data from all centers will be pooled and summarized with respect to demographic and baseline characteristics and efficacy and safety observations. Data will be presented for the complete intent-to-treat population. The primary endpoint will be analyzed in a Cox regression model, with the minimization factors as covariables.

\section{Interim analysis}

An interim analysis will be performed after 50\% (258 patients) of the total required number of patients has been included. The purpose of this interim review is to assess any clinically significant safety concerns and to determine whether there is evidence that up front surgery (treatment A) is clinically or statistically inferior to immediate systemic therapy without up front surgery (treatment B), with respect to the primary endpoint. To control the overall type I error when performing the interim analysis, the Peto approach will be used to ensure an overall Type I error of 5\% [21,22]. A onesided significance level of 0.001 will be used at the interim analysis.

\section{Discussion}

Recent studies on surgery of the breast tumor in patients with primary distant metastatic disease are 
inconclusive regarding the effect of surgery on overall survival. Most indicate that surgical treatment is associated with a significantly improved overall survival [5-12], but some state that this benefit is caused by confounding, induced by the retrospective study designs [15-18]. In order to provide a definite answer with respect to the role of surgery in primary metastatic breast cancer, a prospective randomized controlled trial, the SUBMIT study, is about to be initiated within The Netherlands. If surgery is shown to be associated with improved survival, it would also be interesting to know more about the biological mechanisms which underly the effect. Therefore, research on circulating tumor or endothelial cells in blood, the immune response and the angiogenic potential of metastases will be considered within the trial. A grant application for a side-study, in which circulating tumor cells (CTCs) will be enumerated and characterized for HER2Neu expression and estrogen receptor status, has already been submitted. This side-study would enable us to address the hypothesis that among patients with a HER2Neu negative primary tumor those with HER2Neu positive CTCs have a worse outcome to standard systemic treatment compared to those with HER2Neu negative CTCs. Additionally, the impact of primary tumor resection on CTC numbers will be analyzed.

\section{Funding}

A grant of Sanofi Aventis was used for the initiation of this study.

Funding for the study is provided by the Dutch Cancer Society (Stichting Koningin Wilhelmina Fonds (KWF) voor de Nederlandse Kankerbestrijding). The project number is JBZ 2011-4995. This grant covers the costs for data management of the clinical study and some additional expenses.

\footnotetext{
Acknowledgements

We thank Prof. dr. G. Borm for the statistical assistance, Drs. Frank van Leeuwen and Saskia van Gastel (IKO Trial Office Nijmegen) for their advice regarding data management and the Dutch Breast Cancer Trialists' Group (BOOG) for their support.

There are no financial disclosures from any of the authors.
}

\section{Author details}

${ }^{1}$ Department of Surgery, Máxima Medical Center, Veldhoven, The Netherlands. ${ }^{2}$ Department of Epidemiology, GROW-School for Oncology and Developmental Biology, Maastricht University Medical Centre, Maastricht, The Netherlands. ${ }^{3}$ Division of Medical Oncology, Department Internal Medicine, GROW-School for Oncology and Developmental Biology, Maastricht University Medical Centre, Maastricht, The Netherlands. ${ }^{4}$ Department of Surgery, Jeroen Bosch Hospital, 's-Hertogenbosch, The Netherlands. ${ }^{5}$ Radiotherapeutic Institute Friesland, Leeuwarden, The Netherlands. ${ }^{6}$ Department of Surgical oncology, Dutch Cancer Institute-Antoni van Leeuwenhoek Hospital, Amsterdam, The Netherlands. 'Department of Medical Oncology, VU University Medical Centre, Amsterdam, The Netherlands. ${ }^{8}$ Department of Radiotherapy, Catharina Hospital, Eindhoven,
The Netherlands. ${ }^{9}$ Department of Surgery, Jeroen Bosch Hospital, Postbus 90153, 5200 ME 's-Hertogenbosch, The Netherlands.

\section{Authors' contributions}

All authors made substantive intellectual contributions to the study protocol. They have been involved in drafting the manuscript and read and approved the final version of the manuscript. Conception and design; JR, ME, KB, AV, $V T, E R, E B$. Collection and assembly of data; ME, AV, VT, YL, MS. Manuscript writing; JR, ME, AV, YL, VT, MS. Final approval of manuscript; JR, $M E, K B, A V$, $Y L, V T, E R, E B, M S$.

\section{Competing interests}

The authors declare that they have no competing interests.

Received: 15 September 2011 Accepted: 2 April 2012

Published: 2 April 2012

\section{References}

1. Ruiterkamp J, Ernst MF, de Munck L, van der Heiden-van de Loo M, Bastiaannet E, van de Poll-Franse LV, Bosscha K, Tjan-Heijnen VCG, Voogd AC: Improved survival of patients with primary distant metastatic breast cancer in the period of 1995-2008. A nationwide populationbased study in the Netherlands. Breast Cancer Res Treat 2011, 128:495-503.

2. Ly BH, Nguyen NP, Vinh-Hung V, Rapiti E, Vlastos G: Loco-regional treatment in metastatic breast cancer patients: Is there a survival benefit? Breast Cancer Res Treat 2010, 119:537-545.

3. Chia SK, Speers CH, D'yachkova Y, et al: The impact of new chemotherapeutic and hormone agents on survival in a populationbased cohort of women with metastatic breast cancer. Cancer 2007, 110:973-979.

4. Ernst MF, van de Poll-Franse LV, Roukema JA, et al: Trends in the prognosis of patients with primary metastatic breast cancer diagnosed between 1975 and 2002. Breast 2002, 16:344-351.

5. Khan SA, Stewart AK, Morrow M: Does aggressive local therapy improve survival in metastatic breast cancer? Surgery 2002, 132:620-627.

6. Babiera GV, Rao R, Feng $L$, et al: Effect of primary tumor extirpation in breast cancer patients who present with stage IV disease and an intact primary tumor. Ann Surg Oncol 2005, 13:776-782.

7. Rapiti $E$, Verkooijen HM, Vlastos $G$, et al: Complete excision of primary breast tumor improves survival of patients with metastatic breast cancer at diagnosis. J Clin Oncol 2006, 24:2743-2749.

8. Fields RC, Donna BJ, Trinkaus K, et al: Surgical resection of the primary tumor is associated with increased long-term survival in patients with stage IV breast cancer after controlling for site of metastasis. Ann Surg Oncol 2007, 14(12):3345-3351.

9. Gnerlich J, Jeffe DB, Deshpande AD, Beers C, Zander C, Margenthaler JA Surgical removal of the primary tumor increases overall survival in patients with metastatic breast cancer. Analysis of the 1988-2003 SEER data. Ann Surg Oncol 2007, 14(8):2187-2194.

10. Blanchard DK, Shetty PB, Hilsenbeck SG, Elledge RM: Association of surgery with improved survival in stage IV breast cancer patients. Ann Surg 2008, 247(5):732-738.

11. Ruiterkamp J, Ernst MF, van de Poll-Franse LV, Bosscha K, Tjan-Heijnen VC, Voogd AC: Surgical resection of the primary tumour is associated with improved survival in patients with distant metastatic breast cancer at diagnosis. Eur J Surg Oncol 2009, 35:1146-1151.

12. Neuman HB, Morrogh M, Gonen M, Van Zee KJ, Morrow M, King TA: Stage IV breast cancer in the era of targeted therapy. Does surgery of the primary tumor matter? Cancer 2010, 116(5):1226-1233.

13. Rao R, Feng $L$, Kuerer HM, et al: Timing of surgical intervention for the intact primary in stage IV breast cancer patients. Ann Surg Oncol 2008, 15:1696-1702.

14. Ruiterkamp J, Voogd AC, Bosscha K, Tjan-Heijnen VCG, Ernst MF: Impact of breast surgery on survival in patients with distant metastases at initial presentation. A systematic review of the literature. Breast Cancer Res Treat 2010, 120(1):9-16.

15. Cady B, Nathan NR, Michaelson JS, Golshan M, Smith BL: Matched pair analyses of stage IV breast cancer with or without resection of primary breast site. Ann Surg Oncol 2008, 15:3384-3395.

16. Bafford AC, Burstein HJ, Barkley CR, Smith BL, Lipsitz S, Iglehart JD, Winer EP, Golshan M: Breast surgery in stage IV breast cancer: impact of staging 
and patient selection on overall survival. Breast Cancer Res Treat 2009, 115:7-12.

17. Leung AM, Vu HN, Nguyen KA, Thacker LR, Bear HD: Effects of surgical excision on survival of patients with stage IV breast cancer. J Surg Res 2010, 161(1):83-88.

18. Dominici L, Najita J, Hughes M, Niland J, Marcom P, Wong Y, Carter B, Javid S, Edge S, Burstein H, Golshan Mehra: Surgery of the primary tumor does not improve survival in stage IV breast cancer. Breast Cancer Res Treat 2011.

19. Pocock S, Simon R: Sequential treatment assignment with balancing of prognostic factors in controlled clinical trials. Biometrics 1975, 31:103-115.

20. [http://www.wma.net/en/30publications/10policies/b3/index.html].

21. Haybittle JL: Repeated assessment of results in clinical trials of cancer treatment. Br J Radiol 1971, 44:793-797.

22. Peto R, Pike MC, Armitage $P$, et al: Design and analysis of randomized clinical trials requiring prolonged observation or each patient. $\mathrm{Br} J$ Cancer 1976, 34:585-612.

\section{Pre-publication history}

The pre-publication history for this paper can be accessed here: http://www.biomedcentral.com/1471-2482/12/5/prepub

doi:10.1186/1471-2482-12-5

Cite this article as: Ruiterkamp et al: SUBMIT: Systemic therapy with or without up front surgery of the primary tumor in breast cancer patients with distant metastases at initial presentation. BMC Surgery 2012 12:5.

\section{Submit your next manuscript to BioMed Central} and take full advantage of:

- Convenient online submission

- Thorough peer review

- No space constraints or color figure charges

- Immediate publication on acceptance

- Inclusion in PubMed, CAS, Scopus and Google Scholar

- Research which is freely available for redistribution

Submit your manuscript at www.biomedcentral.com/submit
C Biomed Central 